\title{
SOLVENT SWELLING OF KUKERSITE OIL SHALE MACROMOLECULAR ORGANIC MATTER IN BINARY MIXTURES: IMPACT OF SPECIFICALLY INTERACTING SOLVENTS
}

\author{
JELENA HRULJOVA $^{(a)}$, NATALJA SAVEST ${ }^{(a)}$, \\ ALEXEY YANCHILIN $^{(a)}$, VAHUR OJA $^{(a)^{*}}$, \\ ERIC M. SUUBERG ${ }^{(b)}$
}

(a) Department of Chemical Engineering, Tallinn University of Technology, Ehitajate tee 5, 19086 Tallinn, Estonia

(b) Division of Engineering, Brown University, Providence, RI 02912, USA

\begin{abstract}
The present work deals with the volumetric solvent swelling of the cross-linked macromolecular organic matter (kerogen) of Estonian kukersite oil shale in binary solvent mixtures. A two-step solvent swelling procedure was used in which swelling was performed first in one solvent followed by the addition of a second solvent. The results confirm the important role of specific interactions in determining the swelling behavior of this kerogen. The kerogen swells more in strong electron donor solvents that are able to break specific kerogen-kerogen interaction such as hydrogen bonds. When comparing the maximum swelling achieved, the binary solvent mixtures did not perform any better than strong electron donor solvents, regardless of how the experiments were conducted.
\end{abstract}

Keywords: oil shale, kerogen, kukersite, solvent swelling, binary solvent mixture, electron donor number.

\section{Introduction}

Solvent swelling is a simple and useful technique for determining the solubility parameters of cross-linked polymers, for characterizing solventpolymer interactions, or, using suitable theoretical models, for calculating cross-link densities and molecular weights between cross-links of polymeric networks [1-3]. Volumetric solvent swelling has also been adapted for characterization of complex macromolecular organic structures from solid fossil fuels such as coals and oil shales (for oil shale [4-6]). These studies

\footnotetext{
* Corresponding author: e-mail vahur.oja@ttu.ee
} 
have been mostly aimed at improving the background knowledge of conversion of solid fossil fuels to liquid fuels and energy. While coal has been of continuous commercial interest over the past century, oil shale development has been quite chaotic (with the exception of a few locations such as Estonia, Brazil and China). However lately, due to the increasing search for alternative liquid fuel sources, commercial utilization of oil shale resources has gained more attention. Some general technical characteristics of oil shales and oils can be found from [7-11].

The organic matter of an oil shale consists primarily of a heterogeneous macromolecular structure (called kerogen) and is practically insoluble in organic solvents at room temperature. The yield of solubles, or non-macromolecular extractables, from kukersite oil shale in typical organic solvents does not exceed $1.5 \%$ of the organic matter [12]. Hence, while the extraction processes can influence the thermodynamics of swelling processes (by changing solvent activity), there is not an experimental measurement difficulty caused by significant mass loss during swelling of these materials, if the solvent is changed so as to keep the concentration of extractables low.

Although there have been a considerable number of experimental investigations on oil shale kerogen swelling in single solvents, there are few data found covering swelling in binary solvent mixtures. This paper is an extension of the earlier work from this laboratory [13] and is aimed at giving further insights into the mechanism of solvent swelling of kukersite oil shale kerogen in binary mixtures. This experimental study has been extended to examine the swelling in binary mixtures in two-step swelling procedures in which swelling was performed first in one solvent followed by the addition of a second solvent in order to obtain a desired solvent mixture composition in the swelling tube.

\section{Experimental}

\subsection{Oil shale sample and solvents}

The oil shale kerogen sample used was isolated from kukersite oil shale by a flotation technique [14]. The concentrated sample had a $91 \%$ organic matter content. The elemental composition of the organic matter was $73.3 \mathrm{wt} \% \mathrm{C}$, 8.8 wt.\% H, 1.6 wt.\% N, 16.3 wt. $\% \mathrm{O}+\mathrm{S}$ (by difference), determined using an Exeter Analytical model CE440 elemental analyzer. The samples were ground to a particle size smaller than $100 \mu \mathrm{m}$. All samples were pre-dried 1 hour at $105-110{ }^{\circ} \mathrm{C}$ in air (a standard oil shale drying procedure) before swelling measurements.

The solvents used in preparing the binary solvent mixtures were all reagent grade. Some solvent characteristics of interest in this study are shown in Table, including total (or Hildebrand) solubility parameter $[15,16]$, Gutmann's electron donor (EDN) and electron acceptor (EAN) numbers $[17,18]$, and molar volume [19]. The EDN and EAN are empirical parameters. For example, the EDN is experimentally determined as the negative 
$\Delta \mathrm{H}$-value for the 1:1 adduct formation between $\mathrm{SbCl}_{5}$ (reference) and the solvent molecules in dilute solution of 1,2-dichloroethane [20].

Table. Properties of the solvents used

\begin{tabular}{|l|c|c|c|c|}
\hline \multicolumn{1}{|c|}{ Solvent } & $\begin{array}{c}\text { Solubility parameter } \delta, \\
\mathrm{MPa}^{1 / 2}\end{array}$ & EDN & EAN & $\begin{array}{c}\text { Molar volume } \mathrm{V}_{\mathrm{M}}, \\
\mathrm{cm}^{3} / \mathrm{mol}\end{array}$ \\
\hline Propylamine & 18.2 & $55.5^{\mathrm{a}}$ & $4.8^{\mathrm{a}}$ & 83.0 \\
NMP & 23.1 & 27.3 & 13.3 & 96.5 \\
n-Propanol & 24.5 & 19.8 & 37.7 & 75.2 \\
Benzene & 18.8 & 0.1 & 8.2 & 89.4 \\
Toluene & 18.2 & 0.1 & 3.3 & 106.8 \\
\hline
\end{tabular}

${ }^{a}$ Ethylamine EDN and EAN values were used for propylamine.

${ }^{\mathrm{b}}$ NMP stands for 1-methyl-2-pyrrolidinone.

\subsection{Swelling procedure}

In this study a simple and widely used test tube-based procedure was used that involves solvent swelling of a powdered sample with swollen sample centrifugation. General details of the experimental procedure (test-tube dimensions, centrifugation conditions) are the same as those applied previously for kukersite kerogen swelling in single solvents [21] or in prepared binary mixtures [13].

In the two-stage swelling procedure used in this study the kerogen sample (up to $0.25 \mathrm{~g}$ in the test tube) was first swollen to equilibrium in one solvent. The complete earlier described procedure for swelling in single solvents was followed (for details see [13]). Then, in a second stage, a different solvent was added in the amount needed to obtain the desired final solvent mixture composition. The following two different types of experiments were carried out at this second stage.

In the first, a large excess of the second solvent was added so that after the second stage both solvents were in considerable excess relative to the pure component swelling-related solvent uptake by the kerogen. For this, the solvent above the swollen kerogen sample, swollen to equilibrium in the first solvent, was removed from the tube with a syringe. The amount of solvent left in the swollen sample (between and inside the kerogen particles) was measured gravimetrically. After adding the second solvent the system was mixed well, centrifuged and allowed to swell to a new equilibrium state. The final height of the sample was then measured after allowing 48 hours of swelling in the solvent (for results see section 3.1). Due to experimental limitations (mainly the test tube volume limitation) these experiments were conducted only in the concentration range of the second solvent of 20-80 $\mathrm{mol} \%$.

In the second type of experiment, only a drop of the second solvent was added to the test tube after the first stage (using an automated pipet). The system was then mixed and centrifuged, and the swelling equilibrium was measured after 48 hours. Then the sequence was repeated until the second 
solvent concentration reached almost $5 \mathrm{~mol} \%$, on the basis of total solvents added to the system (for results see section 3.2).

The volumetric swelling was characterized by the usual experimental volumetric swelling extent parameter, the volumetric ratio $Q_{\exp }$, defined as the final swollen volume (or swollen bed height after the second swelling step) divided by the initial unswollen volume (or initial dry bed height) (see [13]). The repeatability of $\mathrm{Q}_{\exp }$ for the two-step procedure used in this study was \pm 0.03 .

The calculation of $\mathrm{Q}_{\exp }$ from experimental data was based on a commonly accepted assumption of a constant void volume fraction, i.e. the void volume fraction in the swollen sample was expected to be equal to that of the initial dry sample. Our latest study on swelling in single solvents suggested that centrifugation caused the swollen sample to be packed more tightly and the actual or true swelling ratio was higher than the one measured by the testtube method [22]. The study also indicated that the extent of compaction depended mostly on the amount of solvent absorbed by the kerogen, and the relationship between the actual swelling ratio and the difference $(\Delta \mathrm{Q})$ followed approximately an exponential trend. Therefore, the following empirical corrective equation is derived from the data of reference [22]: $\mathrm{Q}_{\text {cor }}-\mathrm{Q}_{\exp }=0.0011 \cdot \mathrm{e}^{3.1 \cdot \mathrm{Q}_{\text {cor }}}$. The $\mathrm{Q}_{\text {cor }}$ stands for a corrected, or actual, swelling ratio. In order to get the most reliable fit suitable for the present study, the constants in the equation were obtained by forcing the exponential fit curve to go specifically through the data points for benzene and NMP. Due to the considerable scope of error, the $\mathrm{Q}_{\text {cor }}$ values are shown mainly as an illustration of the consequence of sample compression during centrifugation.

\section{Results and discussion}

In previous studies [21-23] on kukersite oil shale kerogen swelling in single solvents it was observed that the maximum swelling occurred in high electron donor number (EDN) solvents such as propylamine and 1-methyl-2pyrrolidinone (NMP). The molar solvent uptake by kukersite kerogen is shown in Figure 1 as a function of the EDN of solvents used in this study. The value of solvent molar uptake was calculated from $Q_{\exp }$ as $Q_{\exp }-1 / V_{M}$, where $V_{M}$ is the solvent molar volume (for values see Table). The figure shows the same general trend observed previously for kukersite oil shale swelling in 22 pure solvents [21], and emphasizes that high EDN solvents are effective in breaking kerogen-kerogen non-covalent bond interactions (such as hydrogen bonds), resulting in maximum swellability. It also shows that there is a limiting molar uptake of high EDN solvents by the kerogen macromolecular structure (see [24]). 


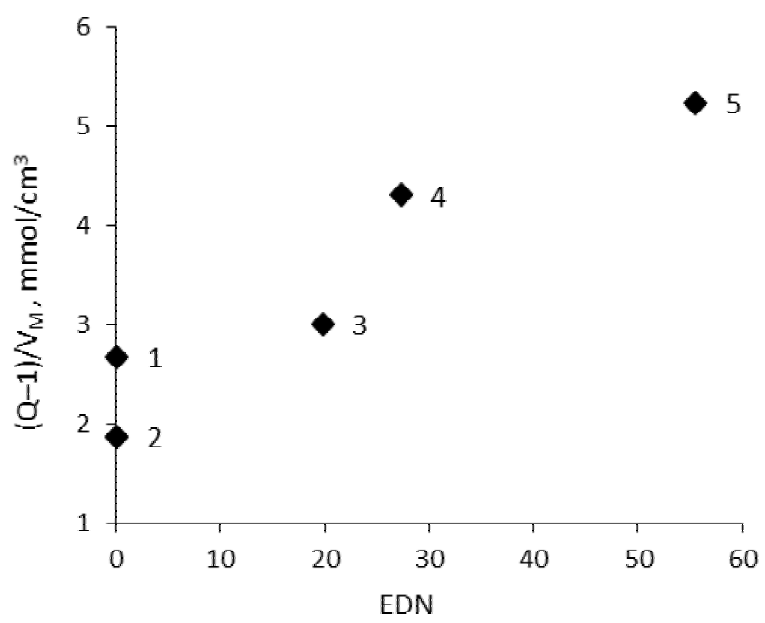

Fig. 1. Variation of solvent molar uptakes with Gutmann's electron donor number (EDN): (1) benzene; (2) toluene; (3) n-propanol; (4) NMP; (5) propylamine (EDN value in this case is taken to be the same as that for ethylamine). The parameter $V_{M}$ is solvent molar volume, as given in Table.

\subsection{Swelling in binary mixtures over the whole concentration range}

Figures 2 to 4 compare the results from the two-stage swelling experiments with those obtained from the earlier reported binary mixture experiments involving direct single-step exposure to pre-prepared binary solvent mixtures (for details see [13]). Open points correspond to experimental Q values (noted as $\mathrm{Q}_{\text {exp }}$ ) measured using the test-tube method and, for illustrative purposes, the solid points show corrected Q values as discussed above (noted as $\mathrm{Q}_{\text {cor }}$ ). Three solvent mixtures (benzene and $\mathrm{n}$-propanol; benzene and NMP; n-propanol and NMP) were selected here because these result in different kerogen swelling behaviors in prepared mixtures, as seen and interpreted in our previous paper [13]. The following is a brief summary of interpretations given in that study [13]: in the case of the pre-prepared benzene-NMP mixture, a small amount of high EDN solvent NMP (less than $10 \mathrm{~mol} \%$ of NMP) increases swelling to approximately the level of pure NMP due to the NMP's ability to reduce apparent cross-link density involving dissociating kerogen-kerogen non-covalent cross-links, i.e. hydrogen bonds. In the case of the pre-prepared benzene-propanol mixture, the swelling behavior can be explained as an interplay between the ability of the weaker (than NMP) donor n-propanol to disrupt kerogen-kerogen noncovalent cross-links and a mixture solubility parameter mismatch. (According to classic thermodynamic swelling theories, the maximum swelling occurs when values of the solubility parameters of the macromolecule and of the solvent mixture do match). In the case of the propanol-NMP mixture, the swelling behavior is believed to be influenced by the ability of n-propanol to act as an electron acceptor $(\mathrm{EAN}=37.7 ; \mathrm{EDN}=19.8)$ relative to the strong electron donor solvent NMP in the mixture. Again, the pre-prepared mixture 


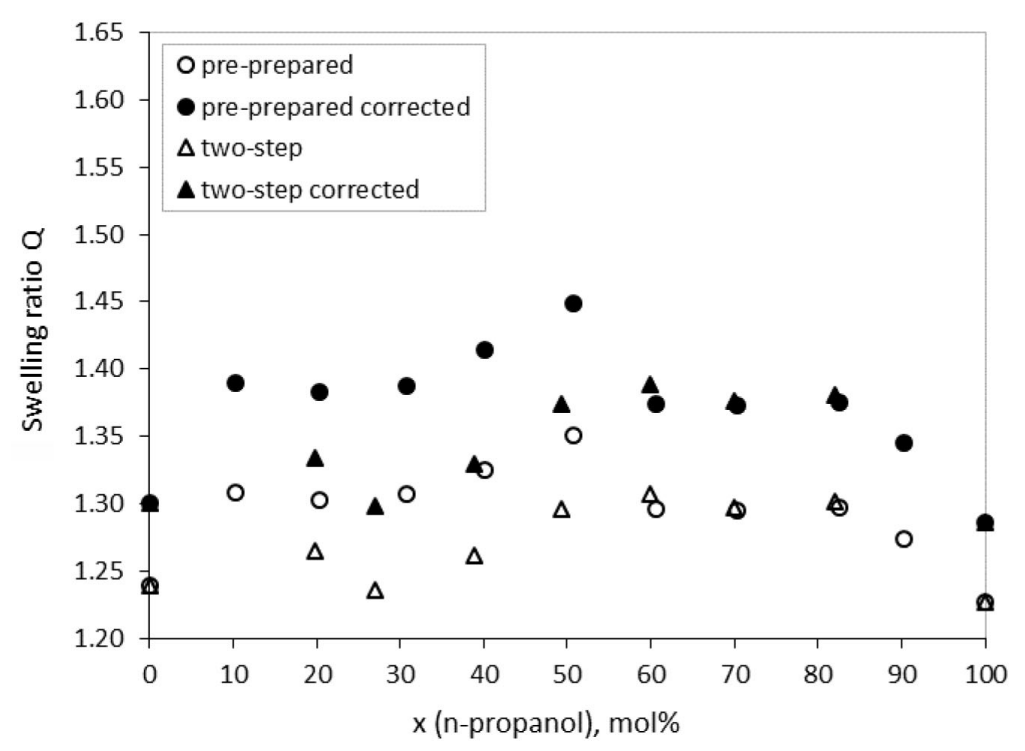

Fig. 2. Kukersite kerogen swelling in benzene and n-propanol mixtures. The open triangles correspond to results from the two-step swelling experiments with benzene as the first solvent. The open circles are for the comparative pre-prepared solvent mixture experiments. The solid points are corrected values for illustrative purposes.

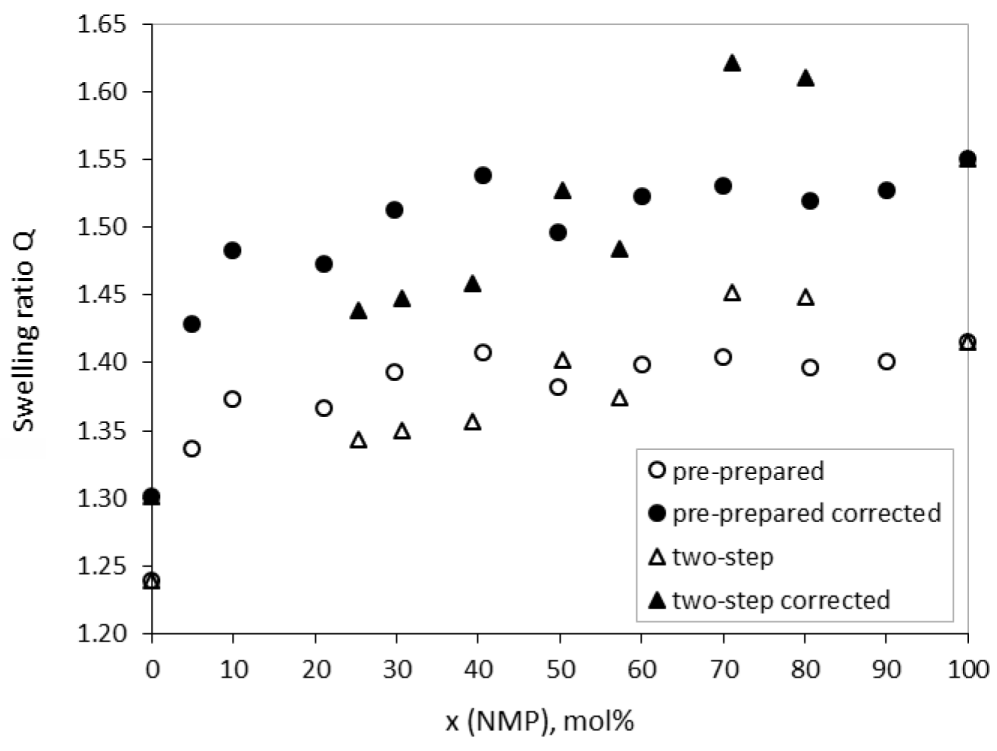

Fig. 3. Kukersite kerogen swelling in benzene and NMP mixtures. The open triangles correspond to the results from the two-step swelling experiments with benzene as the first solvent. The open circles are for the comparative pre-prepared solvent mixture experiments. The solid points are corrected values for illustrative purposes. 


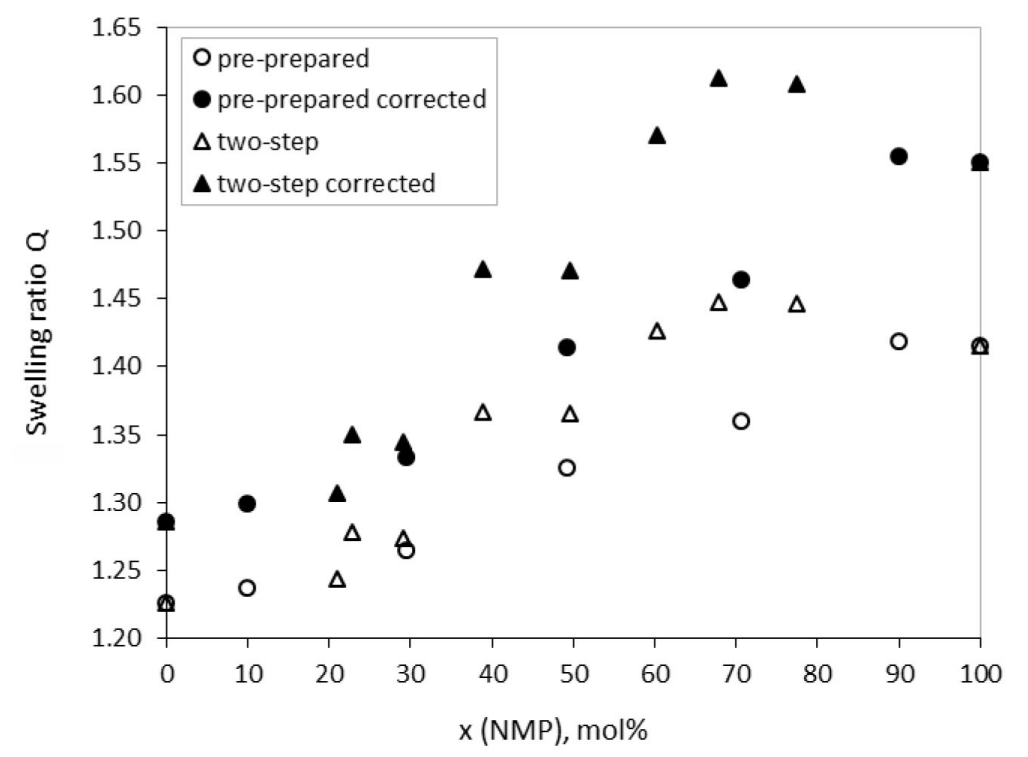

Fig. 4. Kukersite kerogen swelling in n-propanol and NMP mixtures. The open triangles correspond to the results of the two-step swelling experiments with npropanol as the first solvent. The open circles are for the comparative pre-prepared solvent mixture experiments. The solid points are corrected values for illustrative purposes.

swelling experiments were done by the method previously described [13], whereas in the two-stage swelling procedure of interest of this study, the swelling was performed first in one solvent followed by addition of a desired amount of a second solvent achieving a desired solvent mixture composition in the swelling tube. For the two-stage swelling, in the case of benzene-npropanol and benzene-NMP mixtures, benzene was the first solvent added to the tube, and in the case of the n-propanol-NMP mixture, n-propanol was the first added solvent.

Figures 2 to 4 show similarities in the trends in the results from both types of experiments: for the same solvent pairs the swelling behavior of kerogen follows quite similar trends as a function of solvent composition (composition based on solvents added to the test tube). Also, the maximum extent of swelling is comparable, regardless of how the experiments are conducted, though the absolute values from the two types of experiments are often clearly different. Although the differences seen are modest in extent (keeping in mind the \pm 0.03 deviation for two-step swelling and \pm 0.02 for pre-prepared mixture swelling), these results might still be significant in terms of signaling a possible path dependence of the swelling process. For example, Figure 2 shows that when the n-propanol is added after the kerogen has first been swollen in benzene, low concentrations $(<40 \%)$ of n-propanol are not particularly effective in increasing the swelling to values above those for 
benzene by itself, while above $50 \mathrm{~mol} \%$ equivalent in the final mixture of the sequential experiment, the swelling is always comparable to that from the experiment in which the two solvents are added at the same time. This could reflect the fact that the n-propanol cannot be readily absorbed by the benzeneswollen kerogen matrix, until the chemical potential of the propanol exceeds a critical value. There is a large solubility parameter mismatch between these two solvents (see Table), though they are miscible. The observed behavior, in case of which kerogen is more effectively swollen by an equimolar solvent mixture than in the sequential exposure experiments, mirrors other results obtained by swelling coal with methylnaphthalene (a non-specifically interacting solvent, like benzene) and methanol (a specifically interacting solvent, like n-propanol). In these latter experiments, immediate exposure to the mixed solvent resulted in a higher asymptotic swelling than sequential exposure, regardless of which solvent was used for the initial swelling [25]. Such pathdependent swelling behavior is not unique to the cross-linked organic matter of fossil fuels. Such hysteresis-like behavior has been observed in the swelling of cross-linked polystyrene resins in binary solvents [26]. It should be kept in mind that we believe that the results shown in Figure 2 are not kinetically determined, in that enough time has been allowed for the system to reach apparent equilibrium.

On the other hand, it is important to note that, due to kerogen's capabilities to selectively uptake solvent from the solvent mixture [13], it cannot be unequivocally claimed that the solvent compositions shown in Figures 2 to 4 for the two different experiments are identical, insofar as the actual liquid concentrations were not measured here. For example, in one preliminary experiment we determined directly the change in solvent concentration during kukersite swelling in pre-prepared binary mixtures of propanol:benzene (at 10:90) by gas chromatography. The experiment showed that the solvent mixture concentration above the swollen kukersite changed by about $2 \%$ (from 10:90 of propanol:benzene to 8:92), indicating that the estimated solvent composition contained within the swollen network was about 20:80, thereby confirming the existence of selective uptake, even in the case of solvents with weaker donor strength such as propanol $(\mathrm{EDN}=$ 19.8). Thus it is likely that some of differences between the two different types of experiments in Figures 2 through 4 are attributable to differences in uptake of the two components, despite plotting of the points from the two different experiments at the same nominal concentrations. Still, the similarity of the trends with composition are so striking that it is unlikely that differences in amounts of uptake in the two experiments can explain the observed absolute differences.

\subsection{Drop based swelling experiments}

Figure 5 presents the results of experiments in which the second solvent was added by sequential drops to the pre-swollen sample. These experiments are similar to those performed by Larsen et al. on coals [27]. Like the coal 


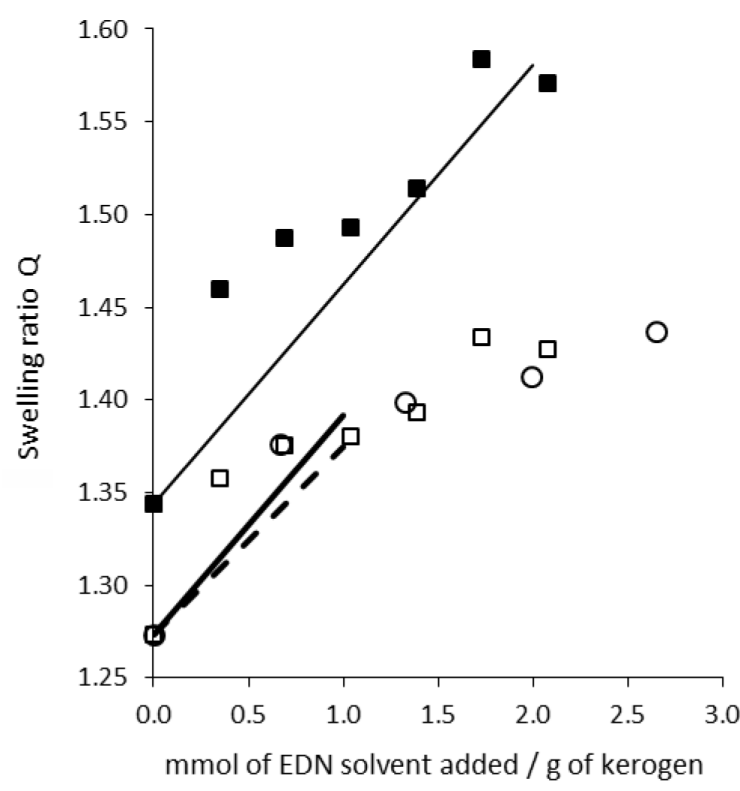

O Toluene + propylamine

$\square \quad$ Toluene + NMP

- Toluene + NMP. Corrected

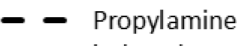
induced swelling

NMP-induced swelling

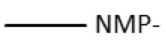

induced. Corrected

Fig. 5. Impact of small amounts of swelling promoting solvents on swelling of kukersite kerogen pre-swollen in toluene. Lines indicate "hypothetical swelling increase" when only all dropwise added strong electron donor solvent (EDN solvent) is absorbed into kerogen structure and causes the extended swelling.

studies, Figure 5 shows swelling ratio profiles where the swelling ratio data are plotted as a function of millimoles of second solvent added per gram of dry kerogen. The non-polar (non-specifically interacting) solvent toluene was chosen as the base solvent, i.e. as the solvent for the first swelling stage. Two different strong EDN solvents, NMP (EDN = 27.3) and propylamine $(\mathrm{EDN}=55.5)$, were used as swelling-promoting solvents in the second stage. While in the first series of experiments described in the previous section, following the second swelling stage both solvents were in excess relative to their kerogen uptake capacities, then here the second solvent, the one added dropwise, can be in deficit relative to the capability of the kerogen to imbibe it.

The two experimental swelling ratio profiles (open squares for NMP and open circles for propylamine as the second solvent) in Figure 5 show similar trends to each other and also qualitatively similar trends to that described by Larsen et al. [27] for coals - addition of small amounts of high EDN solvents can result in increased swelling of a network that was previously in equilibrium with respect to a non-specifically interacting solvent. In the earlier coal studies, this increase of the swelling ratio upon addition of high EDN solvent was explained by the solvent's ability to act as a hydrogen bond (or other strong electron donor-acceptor) breaker. This led to reducing physical macromolecule-macromolecule interactions, reducing effective 
network cross-links and rendering the structure more elastic. In this way, the entropic favorability of solvent imbibition manifests itself, as there is no longer as large an enthalpic penalty for swelling the network structure by greater solvent uptake. The same phenomenon is likely responsible in the system in this study.

Moreover, in the earlier coal studies it was also determined, by gaschromatographic measurements, that all the added high EDN solvent was completely absorbed into the macromolecular swollen coal structure, up to some specific limiting concentration (measured in millimole of high EDN solvent per gram of coal), with the specific concentration depending on the coal used. Assuming that the complete absorption of high EDN solvent can occur also in kukersite kerogen swelling, then the lines in Figure 5 correspond to "hypothetical swelling increase" that could be caused only by absorption of all drop-wise added strong electron donor solvent (NMP - a solid line, propylamine - a dashed line). It can be seen from Figure 5 that initially (below $1 \mathrm{mmol}$ of solvent added per gram of kerogen) the increase in swelling ratio $\left(\mathrm{Q}_{\exp }\right)$ is clearly larger than could be accounted for by uptake of the swelling promoting solvent alone. Again, the open points $\left(\mathrm{Q}_{\exp }\right)$ are the experimentally measured values, and the corresponding lines correspond to a system where no compaction occurs during swollen sample centrifugation. Therefore, for illustrative purposes, the corrected swelling ratios for NMP as a second solvent are also shown as solid points in Figure 5. This comparison illustrates the consequence of the greater elasticity of the swollen network right away at the smallest amounts of added second solvent, as it is network elasticity that also results in greater compaction.

\section{Conclusions}

The observation that significant extents of swelling of kukersite kerogen macromolecular organic matter are achievable only in the presence of strong electron donor number (EDN) solvents, which are able to disrupt specific kerogen-kerogen interactions, such as hydrogen bonds, is supported by the results of this investigation.

Small amounts of specifically interacting high EDN solvents, such as NMP and propylamine, can act as promoters for increased swelling when added to pre-swollen samples in the non-polar (non-specifically interacting) solvent such as toluene. This is consistent with observations from earlier studies on coal macromolecular networks.

Over the whole concentration range, regardless of how the binary mixture based solvent swelling experiments were conducted (using pre-prepared mixtures or the two-step swelling procedure), the swelling behavior and the maximum extents of swelling obtained were comparable. In terms of 
maximizing the swelling, the binary solvent mixtures did not perform any better than pure strong electron donor solvents, such as NMP or propylamine.

\section{Acknowledgement}

The authors gratefully acknowledge financial support provided by the Estonian Ministry of Education and Research under target financing SF0140022s10, and by the graduate school „Functional materials and technologies", which received funding from the European Social Fund under project 1.2.0401.09-0079 in Estonia.

\section{REFERENCES}

1. Flory, P. J. Principles of Polymer Chemistry. Cornell University Press, New York, 1953.

2. Kovac, J. Modified Gaussian model for rubber elasticity. Macromolecules, 1978, 11(2), 362-365.

3. Barr-Howell, B. D., Peppas, N. A. Importance of junction functionality in highly crosslinked polymers. Polym. Bull., 1985, 13(2), 91-96.

4. Larsen, J. W., Li, S. Solvent swelling studies of Green River kerogen. Energ. Fuel., 1994, 8(4), 932-936.

5. Ballice, L. Solvent swelling studies of Göynük (Kerogen Type-I) and Beypazari oil shales (Kerogen Type-II). Fuel, 2003, 82(11), 1317-1321.

6. Kilk, K., Savest, N., Yanchilin, A., Kellogg, D. S., Oja, V. Solvent swelling of Dictyonema oil shale: Low temperature heat-treatment caused changes in swelling extent. J. Anal. Appl. Pyrol., 2010, 89(2), 261-264.

7. Oja, V., Suuberg, E. M. Oil shale processing, chemistry and technology. In: Fossil Energy, Selected Entries from the Encyclopedia of Sustainability Science and Technology (Malhotra, R., ed.). Springer Science + Business Media, New York, 2013, 99-148.

8. Oja, V. Characterization of tars from Estonian Kukersite oil shale based on their volatility. J. Anal. Appl. Pyrol., 2005, 74(1-2), 55-60.

9. Oja, V., Elenurm, A., Rohtla, I., Tali, E., Tearo, E., Yanchilin, A. Comparison of oil shales from different deposits: Oil shale pyrolysis and co-pyrolysis with ash. Oil Shale, 2007, 24(2), 101-108.

10. Urov, K., Sumberg, A. Characteristics of oil shales and shale-like rocks of known deposits and outcrops. Oil Shale, 1999, 16(3 Special: monograph), 1-64.

11. Kelemen, S. R., Afeworki, M., Gorbaty, M. L., Sansone, M., Kwiatek, P. J., Walters, C. C., Freund, H., Siskin, M., Bence, A. E., Curry, D. J., Solum, M., Pugmire, R. J., Vandenbroucke, M., Leblond, M., Behar, F. Direct characterization of kerogen by X-ray and solid-state ${ }^{13} \mathrm{C}$ nuclear magnetic resonance methods. Energ. Fuel., 2007, 21(3), 1548-1561.

12. Koel, M., Ljovin, S., Hollis, K., Rubin, J. Using neoteric solvents in oil shale studies. Pure Appl. Chem., 2001, 73(1), 153-159.

13. Hruljova, J., Savest, N., Oja, V., Suuberg, E. M. Kukersite oil shale kerogen solvent swelling in binary mixtures. Fuel, 2013, 105, 77-82. 
14. Koch, R. P., Kirret, O. G., Oamer, P. E., Ahelik, V. R., Kõrts, A. V. Studies on the flotation process of Estonian kukersite. In: All-Union Conference on the Benefication of Oil Shales. Academy of Sciences USSR, Moscow, 1973, 115122 (in Russian).

15. Karger, B. L., Snyder, L. R., Eon, C. An expanded solubility parameter treatment for classification and use of chromatographic solvents and adsorbents: Parameters for dispersion, dipole and hydrogen bonding interactions. J. Chromatogr. A, 1976, 125(1), 71-88.

16. Belmares, M., Blanco, M., Goddard, W. A., Ross, R. B., Caldwell, G., Chou, S. H., Pham, J., Olofson, P. M., Thomas, C. Hildebrand and Hansen solubility parameters from molecular dynamics with applications to electronic nose polymer sensors. J. Comput. Chem., 2004, 25(15), 1814-1826.

17. Behbehani, G. R., Hamedi, M., Rajabi, F. H. The solvation of urea, tetramethylurea, TMU, in some organic solvents. Int. J. Vib. Spectrosc., 2001, 5(6) [http://www.ijvs.com/volume5/edition6/section3.html].

18. Malavolta, L., Oliveira, E., Cilli, E. M., Nakaie, C. R. Solvation of polymers as model for solvent effect investigation: proposition of a novel polarity scale. Tetrahedron, 2002, 58(22), 4383-4394.

19. Hansen, C. M. Hansen Solubility Parameters: A User's Handbook. CRC Press, 2000.

20. Mayer, U., Gutmann, V., Gerger, W. The acceptor number - A quantitative empirical parameter for the electrophilic properties of solvents. Monatsh. Chem., 1975, 106(6), 1235-1257.

21. Savest, N., Hruljova, J., Oja, V. Characterization of thermally pretreated kukersite oil shale using the solvent-swelling technique. Energ. Fuel., 2009, 23(12), 5972-5977.

22. Hruljova, J., Järvik, O., Oja, V. Application of differential scanning calorimetry to study solvent swelling of kukersite oil shale macromolecular organic matter: A comparison with the fine-grained sample volumetric swelling method. Energ. Fuel., 2014, 28(2), 840-847.

23. Savest, N., Oja, V., Kaevand, T., Lille, Ü. Interaction of Estonian kukersite with organic solvents: A volumetric swelling and molecular simulation study. Fuel, 2007, 86(1-2), 17-21.

24. Suuberg, E. M., Otake, Y., Langner, M. J., Leung, K. T., Milosavljevic, I. Coal macromolecular network structure analysis: solvent swelling thermodynamics and its implications. Energ. Fuel., 1994, 8(6), 1247-1262.

25. Yun, Y., Suuberg, E. M. Cooperative effects in solvent swelling of a bituminous coal. Energ. Fuel., 1998, 12(4), 798-800.

26. O'Kane, J. M., Sherrington, D. C. Hysteresis-like behavior in the swelling/ deswelling of polystyrene cross-linked resins using binary solvent mixtures. Macromolecules, 1990, 23(25), 5286-5291.

27. Larsen, J. W., Gurevich, I., Glass, A. S., Stevenson, D. S. A method for counting the hydrogen-bond cross-links in coal. Energ. Fuel., 1996, 10(6), 12691272.

Received February 5, 2014 